\title{
CT texture based radiomics analysis of Carotid Arteries identifies Vulnerable Patients: a preliminary outcome study.
}

Fulvio Zaccagna, MD, PhD ${ }^{1,2}$, Balaji Ganeshan, $\mathrm{PhD}^{3}$, Marcello Arca, $\mathrm{MD}^{4}$, Marco Rengo, MD, PhD ${ }^{5}$, Alessandro Napoli, MD, PhD ${ }^{2}$, Leonardo Rundo ${ }^{6}$, PhD, Ashley M. Groves, BSc, MBBS, MRCP ${ }^{3}$, Andrea Laghi, MD ${ }^{5}$, lacopo Carbone, MD $^{2}$, Leon J. Menezes, BA BM BCh FRCR FRCP ${ }^{3}$.

\section{Affiliations}

${ }^{1}$ Division of Neuroimaging, Department of Medical Imaging, University of Toronto, Toronto, Canada

${ }^{2}$ Department of Radiological, Oncological and Pathological Sciences, University of Rome - Sapienza, Rome, Italy

${ }^{3}$ Institute of Nuclear Medicine, University College London, London, United Kingdom and NIHR University College London Hospitals Biomedical Research Centre, London, United Kingdom ${ }^{4}$ Internal Medicine Unit, Department of Internal Medicine and Medical Specialties, University of Rome - Sapienza, Rome, Italy

${ }^{5}$ Department of Radiological, Oncological and Pathological Sciences, University of Rome - Sapienza, Polo Pontino, I.C.O.T. Hospital, Latina, Italy

${ }^{6}$ Department of Radiology, University of Cambridge, Cambridge, United Kingdom 


\section{Abstract \\ Purpose}

To assess the potential role of Computed Tomography (CT) texture analysis (CTTA) in identifying vulnerable patients with carotid artery atherosclerosis.

\section{Methods}

In this case-control pilot study, 12 patients with carotid atherosclerosis and a subsequent history of Transient Ischemic Attack or Stroke were age and sex matched with 12 control cases with asymptomatic carotid atherosclerosis (follow-up time $103.58 \pm 9.2$ months).

CTTA was performed using a commercially available research software package (TexRAD) by an operator blinded to clinical data. CTTA comprised a filtrationhistogram technique to extract features at different scales corresponding to spatial scale filter (fine $=2 \mathrm{~mm}$, medium $=3 \mathrm{~mm}$, coarse $=4 \mathrm{~mm}$ ), followed by quantification using histogram-based statistical parameters: mean, kurtosis, skewness, entropy, standard deviation and mean value of positive pixels. A single axial slice was selected to best represent the largest cross-section of the carotid bifurcation on each side.

\section{Results}

CTTA revealed a statistically significant difference in skewness between symptomatic and asymptomatic patients at the medium $(0.22 \pm 0.35$ vs $-0.18 \pm 0.39, p<0.001)$ and coarse $(0.23 \pm 0.22$ vs $0.03 \pm 0.29, p=0.003)$ texture scales. At the fine-texture scale, skewness $(0.20 \pm 0.59$ vs $-0.18 \pm 0.58, p=0.009)$ and standard deviation (366.11 \pm 117.19 vs $300.37 \pm 82.51, p=0.03$ ) were significant before correction. 


\section{Conclusion}

Our pilot study highlights the potential of CTTA to identify vulnerable patients in stroke and TIA. CT texture may have the potential to act as a novel risk stratification tool in patients with carotid atherosclerosis.

\section{Key words}

Computed Tomography Angiography; CTA; Texture Analysis; carotid artery; atherosclerosis; risk stratification. 


\section{Introduction}

Stroke is the second leading cause of death in the developed world and the third worldwide [1]. Carotid artery atherosclerosis is responsible for nearly a third of ischemic strokes [2]. Whereas patients with $70-99 \%$ carotid stenosis can be treated with endarterectomy or stenting [3-5], moderate stenosis is considerably more frequent and accounts for the majority of culprit plaques [6]. Moreover, the management of patients with stenosis $<50 \%$ is even more challenging as there is a subset of patients that develop recurrent neurological symptoms, including stroke [5]. Therefore, there is a need for better risk stratification to ensure optimization of medical therapy or more aggressive surgical or endovascular revascularization [5].

This need has led to the definition of the "vulnerable patient" as a patient with a plaque that yields a high probability of undergoing rapid progression, thus becoming a culprit lesion [7]. Key factors are plaque composition and bio-mechanical forces exercised by blood flow over the plaque itself [7-12]. CT angiography (CTA) is fast, easy and widely available: it is a robust technique to evaluate carotid artery stenosis and plaque morphology $[13,14]$. Several studies have demonstrated that CTA is capable of characterizing plaque composition showing good correlation with histology $[15,16]$.

In the past few years, a method to extract more information related to tissue heterogeneity from standard images has been investigated, namely texture analysis (TA) $[17,18]$. TA refers to a variety of mathematical and statistical techniques that can be used to describe different components of image heterogeneity namely feature/object size, number of features/objects and variation in density of object/feature gray-level intensity in relation to background tissue [17]. To date, the application of TA on CTA has been mostly limited to aortic aneurysm [19-21] with a 
sole pilot study exploring the use of TA in carotid arteries [22]. Therefore, we designed this study to assess the potential role of CT texture analysis (CTTA) in risk stratification of carotid atherosclerosis. The principal hypothesis behind this pilot study is that CT-derived texture of atherosclerotic plaque might be different (heterogeneous, reflecting the underlying distorted micro-architecture) in vulnerable patients whereby texture parameters could provide complementary information to assess risk stratification predicting the potential transformation of a vulnerable plaque into a culprit lesion.

\section{Methods}

Patients

This case-control and cohort exploratory study was based upon retrospective assessment of a dataset of 341 patients with cardiovascular risk factors that underwent Whole-Body CTA (WB-CTA) at our institution as part of previous IRB approved studies [23-25]. The inclusion and exclusion criteria for the WB-CTA are as previously described [23-25]. To identify the patients to be included in this sub analysis, we reviewed the electronic data to firstly select subjects with any carotid plaque and with subsequent development of a transient ischemic attack (TIA) or stroke confirmed by stroke specialist consultant and A\&E admission (flowchart in Figure 1). Patients with posterior circulation stroke were excluded. According to these inclusion criteria, we identified 12 patients ( 7 males; age $63 \pm 10$ years) with carotid atherosclerosis, no history of cerebrovascular incidents preceding the CTA, and a subsequent TIA or stroke; the average time between the CTA and the cardiovascular (CV) events was $22.75 \pm 15.05$ months. Two of those patients who already had a stent in place at the time of CTA were included in this study where the side with the stent 
was excluded from the analysis. These patients were age and sex matched with 12 randomly selected control cases from the whole study population (7 males; age $63 \pm 10$ years) with asymptomatic carotid atherosclerosis (follow-up time $103.58 \pm 9.2$ months). The baseline demographics for both groups are presented in Table 1.

\section{CTA Technique and Data Analysis}

All examinations were carried out on a 64-row CT scanner with z-flying focalspot technology (Somatom Sensation Cardiac 64; Siemens AG; Erlangen, Germany), using the following parameters: $64 \times 0.6 \mathrm{~mm}$ detector configuration, tube potential 100 $\mathrm{kV}$, tube current modulated using the Combined Applications to Reduce Exposure (CARE) Dose 4D system (Siemens Medical Solutions, Forchheim, Germany) with a reference value of $120 \mathrm{mAs}$, pitch 1.2 , gantry rotation time $0.33 \mathrm{~s}$, field of view $28 \mathrm{~cm}$, slice thickness $1 \mathrm{~mm}$, B20 reconstruction algorithm. A high iodine concentration contrast medium (400 mgl/ml; lomeron 400, Bracco, Milan, Italy) was injected through a $18 \mathrm{G}$ cannula inserted into a superficial vein of the antecubital fossa of the right arm using an automatic dual-head injector (Medrad Stellant Dual, Medrad, Palo Alto, PA, USA) with a lodine flux of $1.6 \mathrm{gl} / \mathrm{s}$ followed by a $30 \mathrm{ml}$ saline bolus injected at $4 \mathrm{ml} / \mathrm{s}$. A bolus-tracking technique was used to trigger the acquisition $5 \mathrm{~s}$ after a threshold of 100 Hounsfield units (HU) was reached into the aortic arch.

CTA images were analyzed using a dedicated workstation equipped with software for cardiovascular applications (Aquarius, TeraRecon, San Mateo, CA, USA) as previously described $[23,25]$. Degree of stenosis was assessed using the North American Symptomatic Carotid Endarterectomy Trial (NASCET) method [4]. Plaque composition was assessed using a 3-point Likert scale (1: predominantly calcified; 2 : mixed plaque; 3: predominantly non-calcified). 


\section{CT Texture Analysis}

Texture analysis was performed using TexRAD (Feedback Medical Ltd., https://fbkmed.com/texrad-landing-2/, part of Feedback plc, Cambridge, UK) a proprietary research software algorithm which has undergone an extensive qualification process to assess tumor heterogeneity in radiological images [26-28]. A single operator (BLIND, a radiologist with a subspecialty interest in vascular imaging and 10 years of experience) performed the analysis blinded to the clinical data. All the CTA datasets of the identified subjects were exported in the Digital Imaging and Communications in Medicine (DICOM) format onto an encrypted external hard drive. To avoid bias in analysis related to the knowledge of clinical data, all the identified studies were anonymized, and the code assigned to each patient was recorded in a separate database.

A single axial slice was selected to best represent the largest cross-section of the carotid bifurcation or the greatest degree of stenosis, in presence of an atherosclerotic plaque, bilaterally. A region of interest (ROI) was manually delineated fully enclosing each carotid artery (Figure 2); CTTA was then assessed within this ROI that comprised a filtration-histogram technique. The initial in-plane filtration step comprised a Laplacian of Gaussian (LoG) spatial band-pass filter (similar to a nonorthogonal Wavelet) to produce a series of derived images highlighting features at different anatomic spatial scales ranging from fine-, medium- and coarse-texture. The spatial scale filter (SSF) values ranged between $2-4 \mathrm{~mm}$ in width (i.e. radius) where $\mathrm{SSF}=2 \mathrm{~mm}$ was considered as fine texture scale, $\mathrm{SSF}=3 \mathrm{~mm}$ was considered as medium texture scales, and SSF $=4 \mathrm{~mm}$ was considered as coarse texture scale. Instances with SSF $>4 \mathrm{~mm}$ were not included in the analysis due to the small size of 
the ROls. Heterogeneity was quantified on filtered images using the following histogram based statistical parameters: mean, skewness $(\mathrm{S})$, kurtosis $(\mathrm{K})$, entropy (E), standard deviation (SD) and mean value of positive pixels (MPP) [17]. Extensive validation and a simulation and phantom study by Miles et al clarified what does the filtration-histogram based TA actually mean and how do they reflect different components of heterogeneity[17]. In brief, the mean intensity reflects average brightness, $\mathrm{S}$ reflects asymmetry of the histogram distribution, $\mathrm{K}$ reflects the pointedness or peakedness of the histogram distribution, E reflects irregularity/heterogeneity, SD denotes the width of the histogram or dispersion from the average and the mean of positive pixels reflects the average brightness of only positive pixel values.

\section{Statistical Analysis}

Statistical analysis was performed using dedicated statistical software (SPSS 25.0 statistical package; SPSS Inc, Armonk, NY, USA: IBM Corp.). Continuous values were shown as mean \pm standard deviation ( $\min -\max ; 95 \%$ confidence internal), categorical values were expressed as $\mathrm{n}(\%) \cdot X^{2}$, Student $t$-test and nonparametric Mann-Whitney test were used to assess if there was any significant difference between symptomatic (patients) and asymptomatic (controls) groups. For the statistical analysis of the texture parameters, data from the same subjects were pooled to diminish cluster effect. Statistical significance was set at $5 \%$ for all the performed analyses and corrected for multiple comparisons by using the BenjaminiHochberg procedure, computing the false discovery rate (FDR) at $5 \%$ for all the texture parameters $[29,30]$. Raw $p$ values are shown, and significance is based on Benjamini-Hochberg correction. 
Receiver operating characteristic (ROC) curves were constructed, and the area under the ROC curve (AUC) and diagnostic criteria (optimal cut-off, sensitivity and specificity) was calculated for the texture parameters which showed statistically significant differences between the two groups on the univariate analysis before correction; TIA/stroke was used as the outcome variable to compute the analysis. According to the pilot nature of this study, the optimal cut-off values (i.e. operating point in the ROC curve) were determined favoring sensitivity among specificity, whilst trying to achieve a moderate balance between the two of them.

\section{Results}

We successfully analyzed 46 carotid arteries from the 24 selected patients (14 men, 10 women; patient characteristics in Table 1); two bifurcations were not evaluated due to the presence of stents at the time of the scan and so excluded.

The mean degree of stenosis at the right carotid bifurcation was significantly higher in the patient group compared to the control group (41 \pm 36 vs. $12 \pm 12 ; p=$ 0.01). There was no statistically significant difference in the stenosis degree at the left carotid artery bifurcation (patient group: $12 \pm 16$; control group $17 \pm 22 ; p=0.56$ ) There were no statistically significant differences in plaque composition for both sides (right side $p=0.39$, left side $p=0.72$ ) between the two groups (Table 2).

The comprehensive results of the CTTA are shown in Table 3, Table 4 and Figure 3. Overall, the CTTA revealed a statistically significant difference in $\mathrm{S}$ between the patient group and the control group at the medium and coarse texture scales $(\mathrm{SSF}=3 \mathrm{~mm}$, medium, $0.22 \pm 0.35$ vs $-0.18 \pm 0.39, p<0.001 ;$ and $\mathrm{SSF}=4 \mathrm{~mm}$, coarse, $0.23 \pm 0.22$ vs $0.03 \pm 0.29, p=0.003)$. At the fine texture scale (SSF $=2 \mathrm{~mm})$, 
$S(0.20 \pm 0.59$ vs $-0.18 \pm 0.58, p=0.009)$ and $S D(366.11 \pm 117.19$ vs $300.37 \pm$ 82.51, $p=0.03$ ) were significant before correction.

$\mathrm{ROC}$ analysis was performed for $\mathrm{SD}$ and $\mathrm{S}$ at $\mathrm{SSF}=2$ and $\mathrm{S}$ at $\mathrm{SSF}=3$ and SSF = 4 using TIA/stroke as outcome; the corresponding plots are shown in Figures 4 and 5. Based on the ROC analysis, at $S S F=3$, a $S$ value greater than or equal to 0.015 identified patients with an AUC of 0.81 , sensitivity of $77 \%$ and specificity of $79 \%$ ( $p<0.001$, figure 4). Optimal cut-off, AUC, sensitivity and specificity for $S$ at $\mathrm{SSF}=2$ were respectively $-0.29,0.72,73 \%, 64 \%(p=0.009)$, and at $\mathrm{SSF}=4$ were $0.06,0.76,82 \%, 67 \%(p=0.003)$. AUC values for SD, at SSF $=2$ a value equal or greater than 315.3 was able to identify patients from asymptomatic subjects with an AUC of 0.68 , sensitivity of $64 \%$ and specificity of $71 \%(p=0.033$, figure 5$)$.

\section{Discussion}

In this pilot study, we showed that CTTA parameters, in particular, S and SD, might be able to identify plaques at risk of becoming culprit lesions. We observed that $S$ and SD are the parameters that best correlate with future ischemic events. $S$ is particularly higher in vulnerable patients as compared to asymptomatic control cases. It indicates the presence of hyper-dense objects at any given particular scale [17] which might reflect the presence of microcalcification, micro-vessel proliferation or micro-ulceration within the plaque; the last two being the most expected features. Indeed, ROC analysis showed a high AUC value for $\mathrm{S}$ at all texture scales, i.e., fine-, medium- and coarse-grained texture scales. Although this needs further confirmation, we may envisage the future application of this parameter as a predictor for TIA/stroke episodes. SD at fine-gained texture scale was higher in the patient group compared to controls and could potentially reflect a higher number of hyper-dense objects in the 
patient group [17]. Moreover, provided that acquisition technique, post-processing and iodine delivery rate are constant, texture analysis could also have a role in assessing the heterogeneity within the lumen as a surrogate for flow dynamics. An increase in turbulent flow could potentially cause an increase in entropy. In the current study, we did not see relevant changes in texture parameters that could be attributed to flow dynamics within the lumen, likely due to the low prevalence of severe stenosis, however, we envisage that this information, in particular in patients with severe stenosis, could complement the plaque analysis.

These findings may have a significant impact in routine clinical practice because patients' risk of developing an acute ischemic attack could be better estimated by combining CTTA and current risk stratification tools. At present, the management of patients with stenosis above the threshold proposed by the NASCET and European Carotid Surgery Trial (ECST) studies is well defined, however, lower degree of stenosis still poses a significant challenge [3-5]. Recently, the European Society for Vascular Surgery (ESVS) published a set of guidelines which also aimed at defining treatment for patients with carotid stenosis $<70 \%$ [5]. Medical treatment has been proposed as class I intervention in asymptomatic patients with stenosis $<60 \%$ and in symptomatic patients with stenosis $<50 \%$; however, the working group highlighted the unmet need for clinical/imaging algorithms able to identify a subset of patients who would benefit from a more aggressive approach, with imaging criteria having a predominant role. Nevertheless, the treatment for asymptomatic patients with stenosis $>60 \%$ and symptomatic patients with stenosis $50-69 \%$ is still controversial due to conflicting evidence. Therefore, the potential capability of CTTA to identify plaques at risk of causing a cerebrovascular event despite the degree of 
stenosis, may be particularly useful in guiding/optimizing management in patients with mild to moderate stenosis, currently the most challenging subgroup of patients.

To our knowledge, this pilot study represents the first use of CTTA to investigate the relationship between heterogeneity as assessed by texture analysis of carotid artery plaque and future cardiovascular events in a prospectively enrolled cohort of asymptomatic patients with CV risk factors. Previous studies have proved that CT signal heterogeneity in small abdominal aortic aneurysm (AAA), assessed using CTTA, might be considered as a risk stratification tool to identify untreated aneurysms at risk of significant expansion [20]. CTTA seems to correlate with AAA metabolism as defined by ${ }^{18} \mathrm{~F}$-Fluorodeoxyglucose Positron Emission Tomography [20]. Moreover, CTTA might be a valuable tool to classify aortic abdominal aneurysm evolution after EVAR $[19,21]$. Those studies clearly demonstrated the potential utility of CTTA in cardiovascular imaging as a risk stratification tool and carotid artery imaging is a natural potential application for such an analysis. Acharya et al. explored a potential combination of discrete wavelet transform and texture analysis to build a classifier of CTA images that would be able to identify symptomatic and asymptomatic patients [19]. Despite the promising results, however, the recruited patients were already symptomatic at the time of the analysis and symptomatic plaques have histological distinctive features that clearly separate them from asymptomatic plaques [31]. Therefore, the differences in plaque microarchitecture and composition may have driven the results undermining the prognostic capability of the technique in a routine clinical practice. 
Previous studies explored several different types of texture analysis on ultrasound images of carotid arteries, however, only a few focused on the predictive capability of texture analysis in determining future ischemic events in asymptomatic patients [32-34]. Texture analysis of US images in addition to the clinical characteristics and the degree of stenosis was able to improve the prediction of subsequent ipsilateral cerebrovascular events both using first order statistics [32] and using support vector machines (SVM) [33]. Similarly, a model combining Framingham risk score, total plaque volume and plaque texture of 3D ultrasound images was significantly better than conventional risk stratification approach in predicting future cerebrovascular events [34]. However, in those studies, only approximately $10-11 \%$ of the patient cohort became symptomatic during follow-up.

Our pilot study is a step forward from those previously published work. CT is a more reliable technique, far less operator-dependent than ultrasound and, therefore, more suitable for quantification as a reliable quantitative imaging biomarker and potential adjunct as a risk assessment tool. Moreover, Nyman et al. recently demonstrated that plaque texture analysis of US images is influenced by the cardiac phase at which images are acquired, the size of the plaque and the echogenicity [35]. The effect of the cardiac phase may be explained by the compression and out-ofplane movement of the plaque due to the increased pressure during systole causing significant variation in texture features as also demonstrated by other recent studies $[36,37]$. In addition to the out-of-plane motion, Nyman et al. also highlighted the variability observed in small plaques. The influence of the echogenicity is supposedly due to the higher presence of lipids within echolucent plaques resulting in softer and more elastic plaques more prone to compression and deformation during systole 
which in turn would result in higher ultrasonic reflection. Overall, the variability determined a reclassification of $16-25 \%$ of cases depending on the chosen cutoff values leading the authors to question the possibility of successfully characterize small echolucent plaques. CTA is intrinsically more reproducible than US, less sensitive to motion and the attenuation coefficient is based on the tissue density therefore it is not influenced by the cardiac cycle.

Moreover, in our cohort, all the patients were asymptomatic at the time of the CTA with the events happening an average of 23 months after the examination. Therefore, our technique has been able to correctly predict a future event at a time when no other conventional imaging features would have predicted a future acute ischemic attack. A further significant strength of our pilot study is the perfect match between the asymptomatic and symptomatic group leading to a $50 \%$ of events rate within our cohort. Indeed, as the asymptomatic pool was selected from a larger cohort of patients, we purposely matched the number and characteristics of the two groups to avoid any over- or under- fitting effect potentially introduced in skewed cohorts.

Nevertheless, this current study has several limitations. The first is the small sample size being a pilot study. Although in a small study population, a significant difference in some texture parameters between the symptomatic and control groups were observed. The second most important limitation is that we analyzed both sides due to the retrospective nature of this study and the lack of more detailed clinical information, which did not allow for a clear identification of the future culprit side. However, Rothwell et al. [38] showed that patients with plaque surface irregularity in the symptomatic carotid artery were more likely to have irregularity in the 
contralateral carotid artery as compared to patient with smooth plaque, suggesting a predisposition to plaque irregularity - i.e., texture heterogeneity - and rupture within individuals. This finding combined with the cluster correction we performed before the analysis and application of statistical correction for multiple comparisons (and still the observation of statistically significant differences between the two groups) to certain extent mitigate the limitation of using both sides and the lack of detailed clinical information. Nevertheless, we envisage the need for a larger, prospective study, ideally with pathological confirmation, which will allow for further confirmation of our pilot results. This in-depth evaluation can provide further biologic rationale and correlation between CTTA, symptoms, culprit plaque and outcome.

In conclusion, our pilot study highlights the potential of CTTA to act as a novel non-invasive imaging biomarker to detect vulnerable plaque, independent of the degree of stenosis, in patients with carotid artery atherosclerosis without the need for additional imaging and complex/invasive procedures, with the potential to improve risk stratification and management. 


\section{References}

1. Mortality and Causes of Death Collaborators (2015) Global, regional and national levels of age-specific mortality and 240 causes of death, 1990-2013: A systematic analysis for the Global Burden of Disease Study 2013. Lancet 385:117-71. https://doi.org/10.1016/S0140-6736(14)61682-2

2. Levy El, Mocco J, Samuelson RM, et al (2008) Optimal Treatment of Carotid Artery Disease. J Am Coll Cardiol 51:979-985.

https://doi.org/10.1016/j.jacc.2007.10.052

3. ESCT (1991) MRC European Carotid Surgery Trial: interim results for symptomatic patients with severe (70-99\%) or with mild (0-29\%) carotid stenosis. European Carotid Surgery Trialists' Collaborative Group. Lancet $337: 1235-43$

4. NASCET (1991) Beneficial effect of carotid endarterectomy in sympotamatic patients with high-grade carotid stenosis. N Engl J Med 325:445-453

5. Naylor AR, Ricco JB, de Borst GJ, et al (2018) Editor's Choice - Management of Atherosclerotic Carotid and Vertebral Artery Disease: 2017 Clinical Practice Guidelines of the European Society for Vascular Surgery (ESVS). Eur J Vasc Endovasc Surg 55:3-81. https://doi.org/10.1016/j.ejvs.2017.06.021

6. Barnett JMH, Taylor WD, Eliasziw M, et al (1998) Benefit of carotid endarterectomy in patients with symptomatic moderate or severe stenosis. $\mathrm{N}$ Engl J Med 339:1415-1425

7. Naghavi M, Libby P, Falk E, et al (2003) From Vulnerable Plaque to Vulnerable Patient: A Call for New Definitions and Risk Assessment Strategies: Part I. Circulation 108:1664-1772. https://doi.org/10.1161/01.CIR.0000087481.55887.C9 
8. Teng Z, Sadat U, Brown AJ, Gillard JH (2014) Plaque hemorrhage in carotid artery disease: Pathogenesis, clinical and biomechanical considerations. J Biomech 47:847-858. https://doi.org/10.1016/j.jbiomech.2014.01.013

9. Stary HC (2000) Natural history and histological classification of atherosclerotic lesions: an update. Arterioscler Thromb Vasc Biol 20:1177-8

10. Stary HC, Chandler AB, Dinsmore RE, et al (1995) A definition of advanced types of atherosclerotic lesions and a histological classification of atherosclerosis. A report from the Committee on Vascular Lesions of the Council on Arteriosclerosis, American Heart Association. Circulation 92:135574

11. Dweck MR, Maurovich-Horvat P, Leiner T, et al (2020) Contemporary rationale for non-invasive imaging of adverse coronary plaque features to identify the vulnerable patient: a Position Paper from the European Society of Cardiology Working Group on Atherosclerosis and Vascular Biology and the European Associa. Eur Hear J - Cardiovasc Imaging 1-7. https://doi.org/10.1093/ehjci/jeaa201

12. Sillesen H, Sartori S, Sandholt B, et al (2018) Carotid plaque thickness and carotid plaque burden predict future cardiovascular events in asymptomatic adult Americans. Eur Heart J Cardiovasc Imaging 19:1042-1050. https://doi.org/10.1093/ehjci/jex239

13. Anzidei M, Napoli A, Zaccagna F, et al (2012) Diagnostic accuracy of colour Doppler ultrasonography, CT angiography and blood-pool-enhanced MR angiography in assessing carotid stenosis : a comparative study with DSA in 170 patients. Radiol Med 117:54-71. https://doi.org/10.1007/s11547-011-0651- 
14. Chappell FM, Wardlaw JM, Young GR, et al (2009) Carotid artery stenosis: accuracy of noninvasive tests--individual patient data meta-analysis. Radiology 251:493-502. https://doi.org/10.1148/radiol.2512080284

15. Wintermark M, Jawadi SS, Rapp JH, et al (2008) High-resolution CT imaging of carotid artery atherosclerotic plaques. AJNR Am J Neuroradiol 29:875-882. https://doi.org/10.3174/ajnr.A0950

16. De Weert TT, Ouhlous M, Meijering E, et al (2006) In vivo characterization and quantification of atherosclerotic carotid plaque components with multidetector computed tomography and histopathological correlation. Arterioscler Thromb Vasc Biol 26:2366-2372.

https://doi.org/10.1161/01.ATV.0000240518.90124.57

17. Miles KA, Ganeshan B, Hayball MP (2013) CT texture analysis using the filtration-histogram method: what do the measurements mean? Cancer Imaging 13:400-6. https://doi.org/10.1102/1470-7330.2013.9045

18. Bharati MH, Liu JJ, MacGregor JF (2004) Image texture analysis: methods and comparisons. Chemom Intell Lab Syst 72:57-71. https://doi.org/10.1016/j.chemolab.2004.02.005

19. García G, Maiora J, Tapia A, De Blas M (2012) Evaluation of texture for classification of abdominal aortic aneurysm after endovascular repair. J Digit Imaging 25:369-76. https://doi.org/10.1007/s10278-011-9417-7

20. Kotze CW, Rudd JHF, Ganeshan B, et al (2014) CT signal heterogeneity of abdominal aortic aneurysm as a possible predictive biomarker for expansion. Atherosclerosis 233:510-7.

https://doi.org/10.1016/j.atherosclerosis.2014.01.001

21. Ding N, Hao Y, Wang Z, et al (2020) CT texture analysis predicts abdominal 
aortic aneurysm post-endovascular aortic aneurysm repair progression. Sci Rep 10:1-10. https://doi.org/10.1038/s41598-020-69226-1

22. Acharya UR, Sree SV, Mookiah MRK, et al (2013) Computed tomography carotid wall plaque characterization using a combination of discrete wavelet transform and texture features: A pilot study. Proc Inst Mech Eng Part H J Eng Med 227:643-654. https://doi.org/10.1177/0954411913480622

23. Napoli A, Anzidei M, Francone M, et al (2008) 64-MDCT imaging of the coronary arteries and systemic arterial vascular tree in a single examination: optimisation of the scan protocol and contrast-agent administration. Radiol Med 113:799-816. https://doi.org/10.1007/s11547-008-0304-3

24. Arca M, Pigna G, Zaccagna F, et al (2010) Atherosclerotic burden In asymptomatic patients with metabolic syndrome evaluated by computed tomography angiography. Atheroscler Suppl 11:18.

https://doi.org/10.1016/S1567-5688(10)70076-3

25. Pigna G, Napoli A, Zaccagna F, et al (2011) The relationship between metabolic syndrome, its components, and the whole-body atherosclerotic disease burden as measured by computed tomography angiography. Atherosclerosis 215:417-420

26. Ganeshan B, Panayiotou E, Burnand K, et al (2012) Tumour heterogeneity in non-small cell lung carcinoma assessed by CT texture analysis: a potential marker of survival. Eur Radiol 22:796-802. https://doi.org/10.1007/s00330-0112319-8

27. Goh V, Ganeshan B, Nathan P, et al (2011) Assessment of response to tyrosine kinase inhibitors in metastatic renal cell cancer: CT texture as a predictive biomarker. Radiology 261:165-71. 
https://doi.org/10.1148/radiol.11110264

28. Miles KA, Ganeshan B, Griffiths MR, et al (2009) Colorectal cancer: texture analysis of portal phase hepatic CT images as a potential marker of survival. Radiology 250:444-52. https://doi.org/10.1148/radiol.2502071879

29. Benjamini $Y$, Hochberg $Y(1995)$ Controlling the False Discovery Rate : A Practical and Powerful Approach to Multiple Testing. J R Stat Soc 57:289-300

30. McDonald JH (2014) Handbook of Biological Statistics, 3rd ed. Sparky House Publishing, Baltimore, Maryland

31. Golledge J, Greenhalgh RM, Davies AH (2000) The Symptomatic Carotid Plaque. $774-781$

32. Nicolaides AN, Kakkos SK, Kyriacou E, et al (2010) Asymptomatic internal carotid artery stenosis and cerebrovascular risk stratification. J Vasc Surg 52:1486-1496.e5. https://doi.org/10.1016/j.jvs.2010.07.021

33. Kyriacou EC, Petroudi S, Pattichis CS, et al (2012) Prediction of high-risk asymptomatic carotid plaques based on ultrasonic image features. IEEE Trans Inf Technol Biomed 16:966-973. https://doi.org/10.1109/TITB.2012.2192446

34. Engelen A van, Wannarong T, Parraga G, et al (2014) Three-dimensional carotid ultrasound plaque texture predicts vascular events. Stroke 45:26952701. https://doi.org/10.1161/STROKEAHA.114.005752

35. Nyman E, Lindqvist $P$, Näslund U, Grönlund C (2018) Risk Marker Variability in Subclinical Carotid Plaques Based on Ultrasound is Influenced by Cardiac Phase, Echogenicity and Size. Ultrasound Med Biol 44:1742-1750. https://doi.org/10.1016/j.ultrasmedbio.2018.03.013

36. Loizou CP, Pattichis CS, Pantziaris M, et al (2016) Texture features variability in ultrasound video of atherosclerotic carotid plaques. IFMBE Proc 57:351- 
354. https://doi.org/10.1007/978-3-319-32703-7_69

37. Kyriacou E, Christofides S, Pattichis CS (2016) A Mathematical Model of Discharge Coefficient for Prosthetic Valves' Performance Evaluation. IFMBE Proc 57:688-693. https://doi.org/10.1007/978-3-319-32703-7

38. Rothwell PM, Villagra R, Gibson R, et al (2000) Evidence of a chronic systemic cause of instability of atherosclerotic plaques. Lancet 355:19-24.

https://doi.org/10.1016/S0140-6736(99)04470-0 


\section{Tables}

\begin{tabular}{|c|c|c|c|}
\hline & $\begin{array}{l}\text { Symptomatic Group } \\
(n=12)\end{array}$ & $\begin{array}{l}\text { Asymptomatic Group } \\
(n=12)\end{array}$ & $p$ \\
\hline Age (years) & $63 \pm 10$ & $63 \pm 10$ & 0.98 \\
\hline Male, n (\%) & $7(58.3)$ & $7(58.3)$ & 1.00 \\
\hline CV risk factors $(n)$ & $3.83 \pm 1.5$ & $3.17 \pm 2.0$ & 0.37 \\
\hline Diabetes & $5(41.7)$ & $2(16.7)$ & 0.19 \\
\hline Smoking & $2(16.7)$ & $4(33.3)$ & 0.31 \\
\hline Hypertension & $11(91.7)$ & $7(58.3)$ & 0.06 \\
\hline Dyslipidemia & $9(75)$ & $5(41.7)$ & 0.10 \\
\hline Body Mass index & $28.7 \pm 3.2$ & $28.2 \pm 3.0$ & 0.62 \\
\hline Family history of HLP & $5(41.7)$ & $5(41.7)$ & 1.00 \\
\hline Family history of CVD & $6(50)$ & $8(66.7)$ & 0.42 \\
\hline
\end{tabular}

Therapy

$\begin{array}{llll}- \text { Antihypertensive } & 9(75) & 7(58.3) & 0.42 \\ \text { - Hypoglycemic } & 5(41.7) & 2(16.7) & 0.20 \\ \text { - Lipid-lowering } & 5(58.3) & 4(33.3) & 0.62\end{array}$


Table 1: Demographic table.

Continuous values are expressed as mean $\pm \mathrm{sd}$, categorical data as natural numbers (\%). Cardiovascular risk factors are listed individually and the sum of risk factors in each patient is provided as "CV risk factors $(n)$. Therapy refers to the treatment the patients were undergoing at the time of recruitment. Abbreviations: $\mathrm{CV}=$ cardiovascular, HLP = Hyperlipidemia, CVD = cardiovascular disease 


\begin{tabular}{|c|c|c|c|c|c|c|}
\hline \multirow[b]{3}{*}{ Stenosis } & \multicolumn{2}{|c|}{ Right Carotid Bifurcation } & & \multicolumn{2}{|c|}{ Left Carotid Bifurcation } & \multirow[b]{2}{*}{$p$} \\
\hline & $\begin{array}{l}\text { Symptomatic } \\
\text { Group }\end{array}$ & $\begin{array}{l}\text { Asymptomatic } \\
\text { Group }\end{array}$ & $p$ & $\begin{array}{l}\text { Symptomatic } \\
\text { Group }\end{array}$ & $\begin{array}{l}\text { Asymptomatic } \\
\text { Group }\end{array}$ & \\
\hline & $41 \pm 36$ & $12 \pm 12$ & $0.01^{*}$ & $12 \pm 16$ & $17 \pm 22$ & 0.56 \\
\hline \multicolumn{7}{|l|}{ Plaque composition } \\
\hline & & & 0.39 & & & 0.72 \\
\hline No plaque & $2(16.7)$ & $3(25)$ & & $6(50)$ & $5(41.7)$ & \\
\hline Predominantly calcified & $2(16.7)$ & $1(8.3)$ & & - & - & \\
\hline Mixed plaque & $6(50)$ & $2(16.7)$ & & $5(41.7)$ & $6(50)$ & \\
\hline Predominantly non calcified & $2(16.7)$ & $6(50)$ & & $1(8.3)$ & $1(8.3)$ & \\
\hline
\end{tabular}

Table 2: Plaque characteristics.

Continuous values are expressed as mean $\pm \mathrm{sd}$, categorical data as natural numbers (\%). * and bold denotes statistical significance. 


\begin{tabular}{|c|c|c|c|c|c|c|c|c|c|c|c|c|}
\hline & \multicolumn{6}{|c|}{ Symptomatic Group } & \multicolumn{6}{|c|}{ Asymptomatic Group } \\
\hline Filter & Mean & Skewness & Kurtosis & Entropy & SD & MPP & Mean & Skewness & Kurtosis & Entropy & SD & MPP \\
\hline \multirow{2}{*}{ SSF2 } & 150.72 & 0.20 & -0.36 & 5.21 & 366.11 & 392.50 & 158.90 & -0.18 & -0.18 & 5.28 & 300.37 & 322.54 \\
\hline & \pm 93.72 & \pm 0.59 & \pm 1.05 & \pm 0.22 & \pm 117.19 & \pm 132.90 & \pm 40.8 & \pm 0.58 & \pm 1.66 & \pm 0.17 & \pm 82.51 & \pm 72.34 \\
\hline \multirow{2}{*}{ SSF3 } & 267.89 & 0.22 & -0.84 & 5.20 & 404.63 & 480.56 & 269.10 & -0.18 & -0.76 & 5.30 & 330.59 & 418.04 \\
\hline & \pm 145.97 & \pm 0.35 & \pm 0.41 & \pm 0.22 & \pm 137.87 & \pm 176.38 & \pm 61.22 & \pm 0.39 & \pm 0.70 & \pm 0.16 & \pm 73.10 & \pm 77.03 \\
\hline \multirow[t]{2}{*}{ SSF4 } & & & & & & & & & & & & \\
\hline & \pm 181.52 & \pm 0.22 & \pm 0.22 & \pm 0.23 & \pm 144.85 & \pm 197.81 & \pm 80.10 & \pm 0.29 & \pm 0.32 & \pm 0.16 & \pm 66.65 & \pm 83.40 \\
\hline
\end{tabular}

Table 3: Texture parameters.

Continuous values are expressed as mean $\pm \mathrm{sd}$. 
Symptomatic group vs asymptomatic group

\begin{tabular}{lllllll}
\hline Filter & Mean & SD & Entropy & MPP & Skewness & Kurtosis \\
SSF2 & 0.367 & 0.033 & 0.311 & 0.050 & 0.009 & 0.56 \\
SSF3 & 0.482 & 0.065 & 0.262 & 0.312 & $<\mathbf{0 . 0 0 1 ^ { * }}$ & 0.939 \\
& & & & & & \\
SSF4 & 0.629 & 0.367 & 0.166 & 0.826 & $\mathbf{0 . 0 0 3}^{*}$ & 0.214 \\
& & & & & & \\
\hline
\end{tabular}

Table 4: Comparison between symptomatic group and asymptomatic group.

Values are $p$ obtained using the Mann-Whitney test as stated in the statistical analysis paragraph. ${ }^{*}$ and bold denotes statistical significance after correction for multiple comparisons by using the Benjamini-Hochberg procedure with a false discovery rate $(\mathrm{FDR})$ at $5 \%$. 


\section{Figures}

Fig. 1 Patients selection. Flow-chart shows the process used to select the two groups evaluated in this study. The original population was composed by 341 subjects that underwent WB-CTA for previous studies $(24,25)$. We firstly screened the population to highlight subjects with atherosclerotic disease at the carotid artery. Therefore, we included in the symptomatic group patients with a subsequent TIA or stroke recorded during the follow-up. From the asymptomatic pool, we randomly selected sex and age matched controls.

Fig. 2 Example of CTTA at SSF=2 (fine-texture-map) superimposed onto the CTA image in an asymptomatic subject (a) and in a patient with TIA (b).

Fig. 3 Texture parameters in Table 3, graphically represented as mean and standard deviation by the error bars, for both symptomatic and asymptomatic groups, as well as for the three spatial scale filters (SSFs) considered. Statistical significance is denoted as * (according to the $p$ values computed in Table 4).

Fig. 4 ROC curves for the CTTA (skewness). ROC curve highlights the ability of Skewness to distinguish between symptomatic and asymptomatic patients. Texture filter scales comprised SSF=2 (A), SSF=3 (B) and SSF=4 (C). The corresponding AUCs are $0.72(95 \% \mathrm{Cl} 0.576-0.871 ; p=0.009)$ for $\mathrm{SSF}=2 ; 0.81(95 \% \mathrm{Cl} 0.678-$ $0.937 ; p=.0001)$ for $\mathrm{SSF}=3$ and $0.76(95 \% \mathrm{Cl} 0.6100 .903 ; p=0.003)$ for $\mathrm{SSF}=4$. Optical cut offs, sensitivity and specificity are, respectively, $-0.29,73 \%, 64 \%$ at $S S F=2,0.015,77 \%$ and $79 \%$ at $S S F=3$, and $0.06,82 \%, 67 \%$ at $S S F=4$. Diagonal segments are produced by ties.

Fig. 5 SD ROC curve for the CTTA (SD at SSF=2). ROC curve highlights the ability of $\mathrm{SD}$ at $\mathrm{SSF}=2$ to distinguish between symptomatic and asymptomatic patients. The 
corresponding AUC is $0.68(95 \% \mathrm{Cl} 0.527-0.840 ; p=0.033)$; optimal cut off is 315.3 , sensitivity $64 \%$ and specificity $71 \%$. Diagonal segments are produced by ties. 


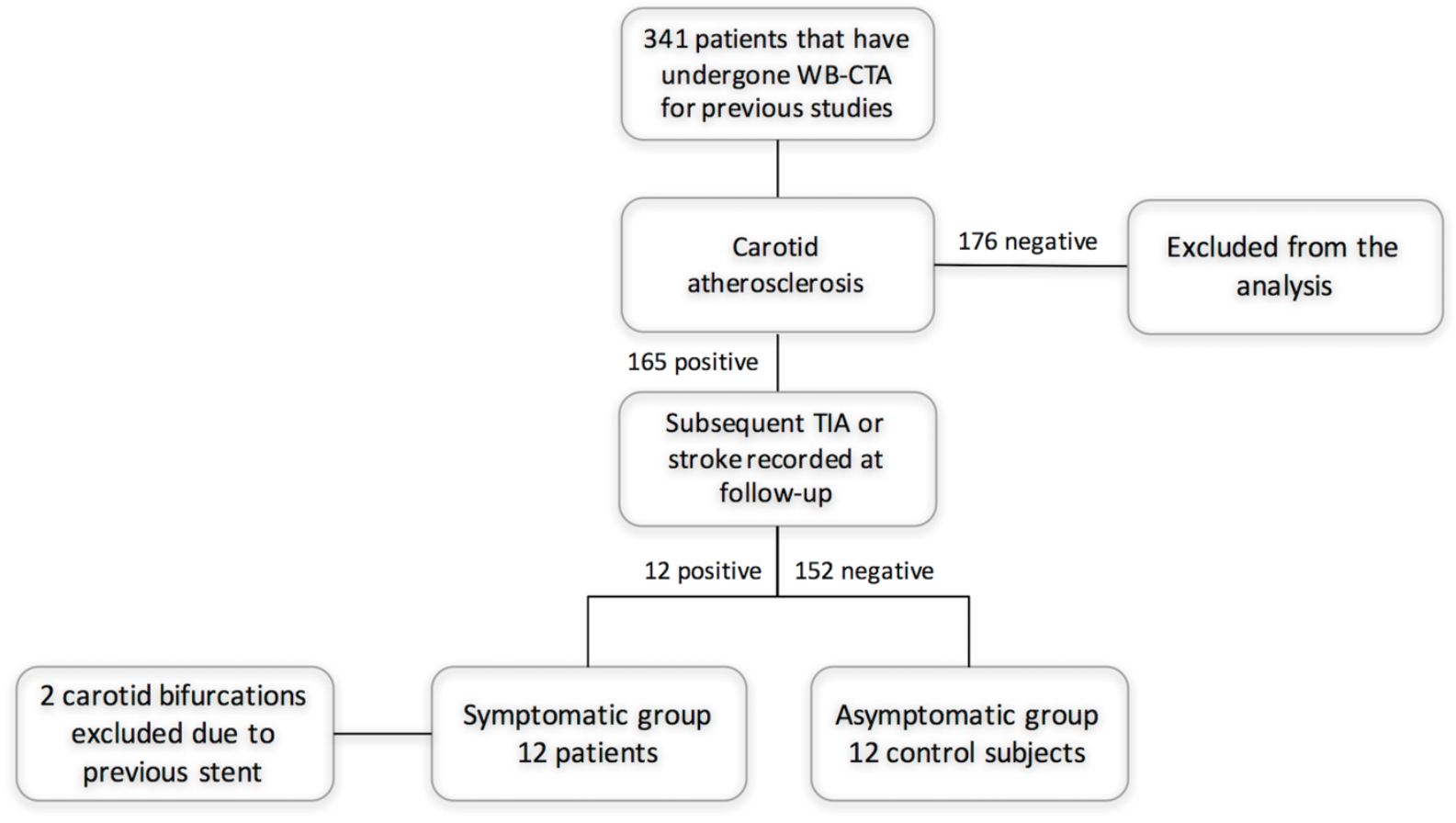

Figure 1 


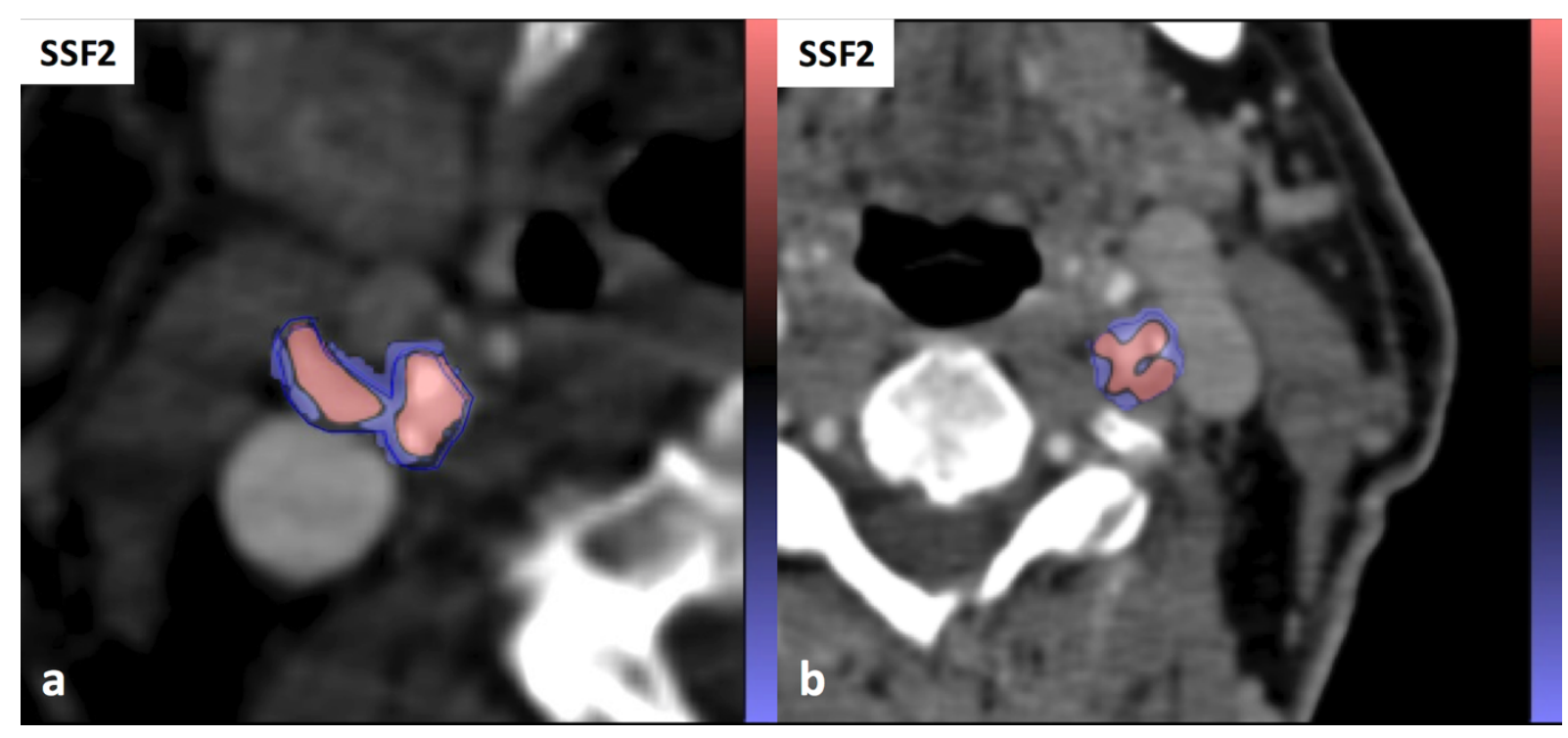

Figure 2 

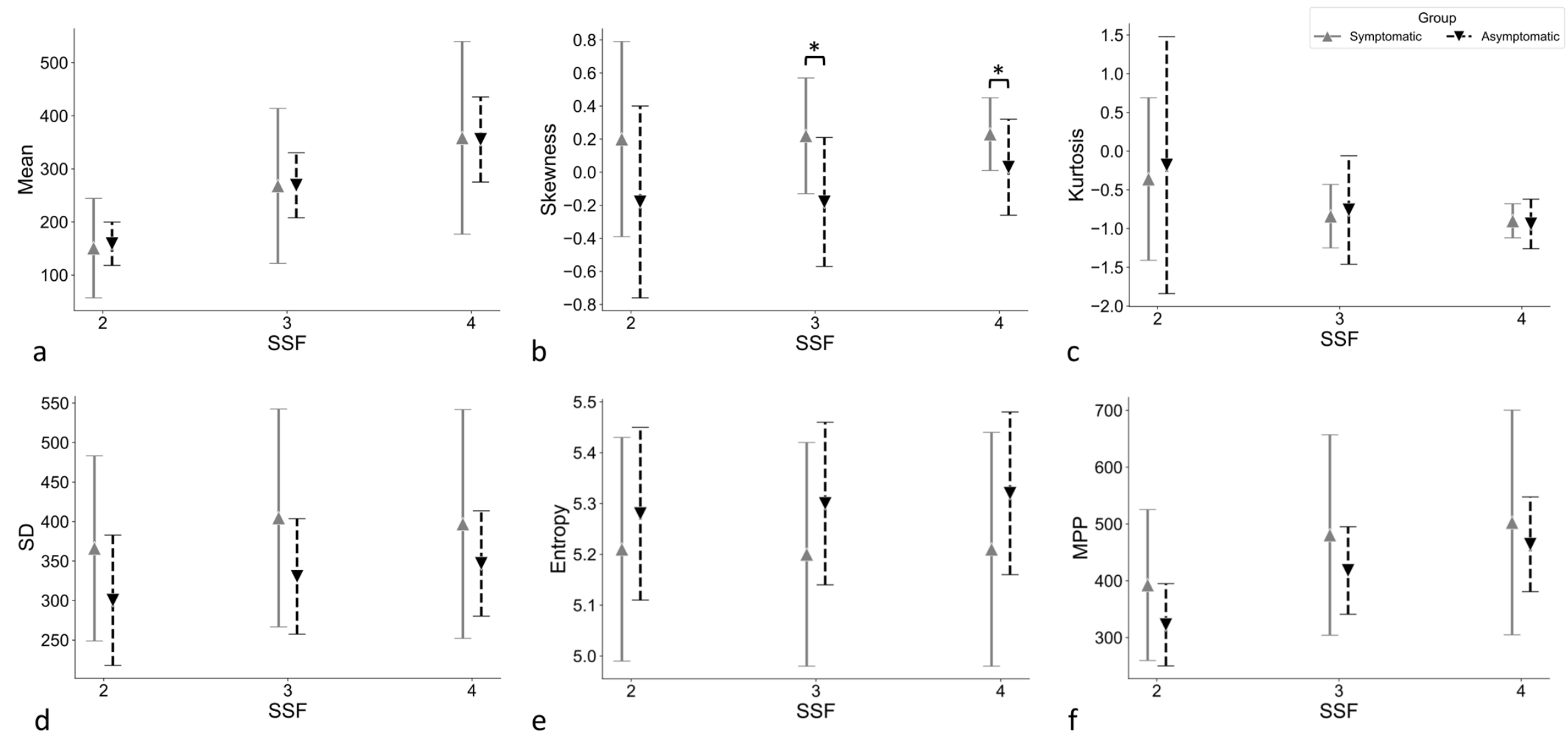

Figure 3 
a

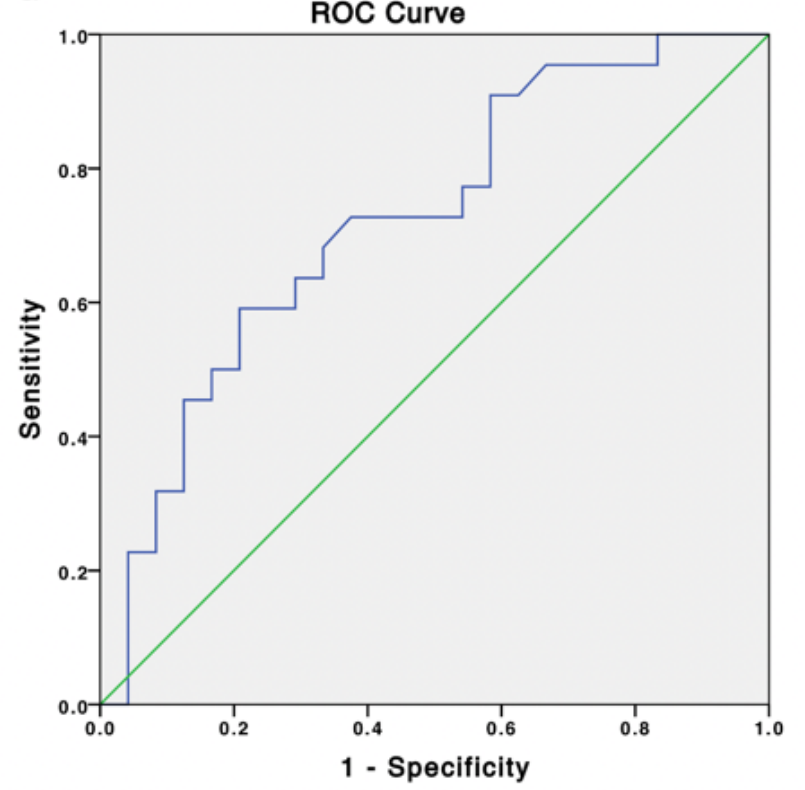

b

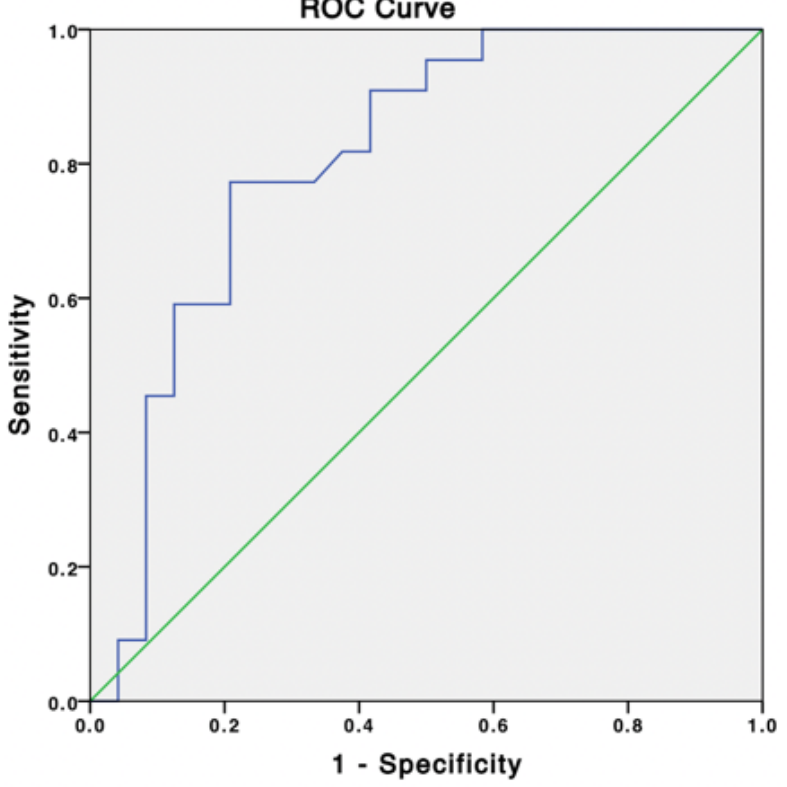

C

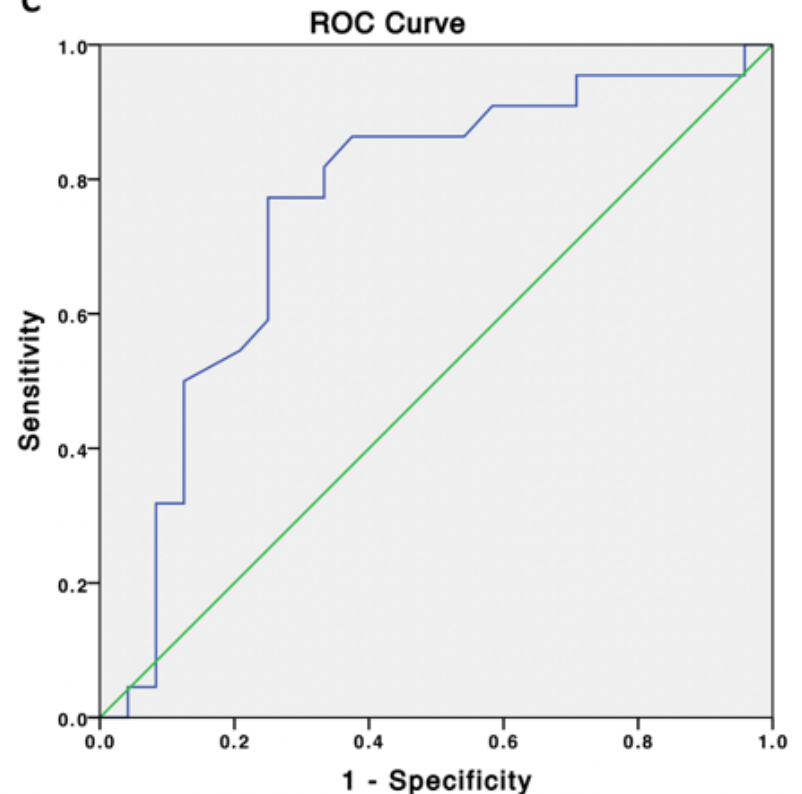

Figure 4 


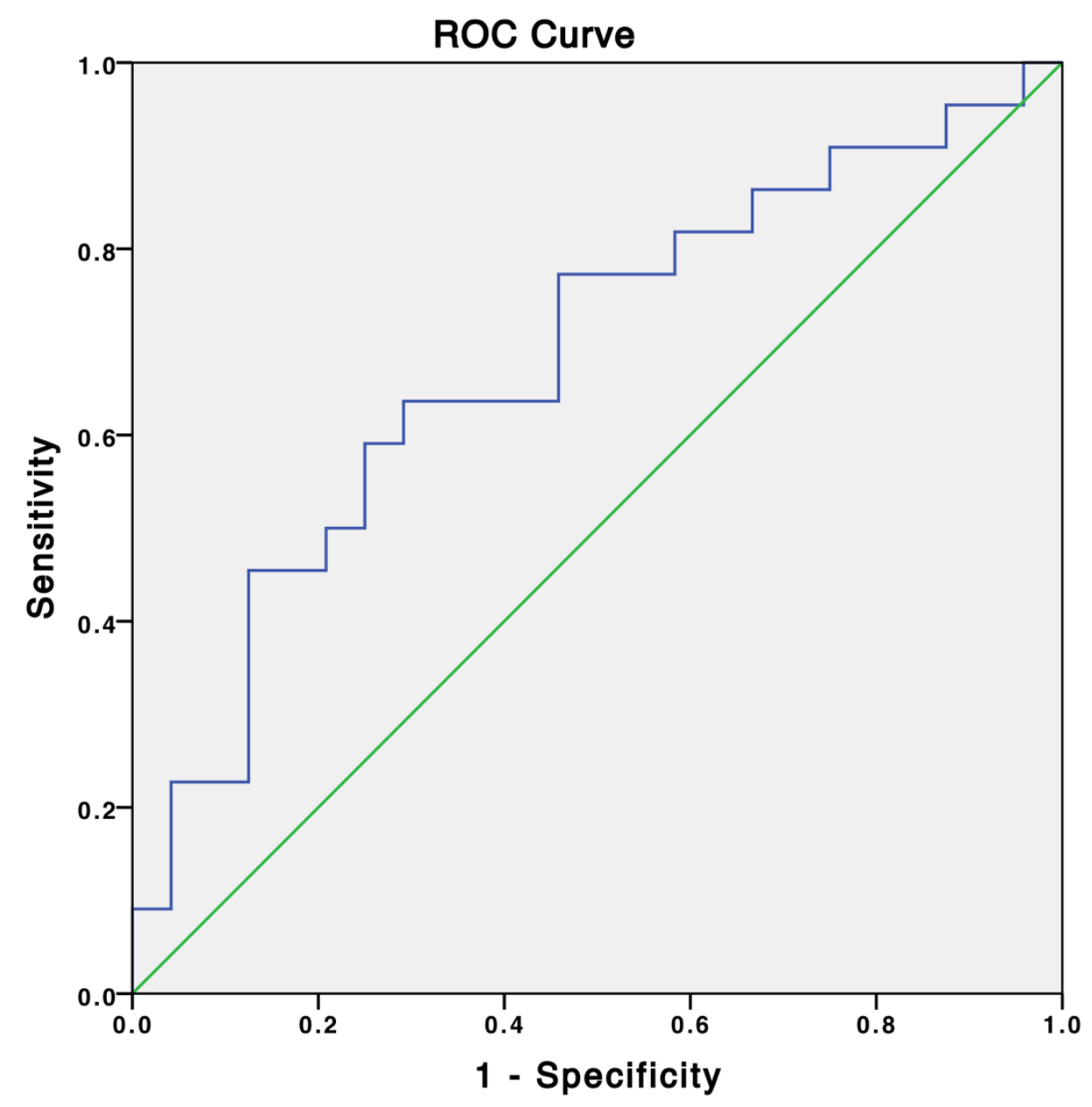

Figure 5 